\title{
Optimization-Based Control
}

\author{
Richard M. Murray \\ Control and Dynamical Systems \\ California Institute of Technology
}

DRAFT v2.1a, January 3, 2010

(c) California Institute of Technology

All rights reserved.

This manuscript is for review purposes only and may not be reproduced, in whole or in part, without written consent from the author. 


\title{
Chapter 3 Receding Horizon Control
}

\author{
(with J. E. Hauser and A. Jadbabaie)
}

This set of notes builds on the previous two chapters and explores the use of online optimization as a tool for control of nonlinear control. We begin with a high-level discussion of optimization-based control, refining some of the concepts initially introduced in Chapter 1. We then describe the technique of receding horizon control (RHC), including a proof of stability for a particular form of receding horizon control that makes use of a control Lyapunov function as a terminal cost. We conclude the chapter with a detailed design example, in which we can explore some of the computational tradeoffs in optimization-based control.

Prerequisites. Readers should be familiar with the concepts of trajectory generation and optimal control as described in Chapters 1 and ??. For the proof of stability for the receding horizon controller that we present, familiarity with Lyapunov stability analysis at the level given in ÅM08, Chapter 4 (Dynamic Behavior) is assumed.

The material in this chapter is based on part on joint work with John Hauser and Ali Jadbabaie $\left[\mathrm{MHJ}^{+} 03\right]$.

\subsection{Optimization-Based Control}

Optimization-based control refers to the use of online, optimal trajectory generation as a part of the feedback stabilization of a (typically nonlinear) system. The basic idea is to use a receding horizon control technique: a (optimal) feasible trajectory is computed from the current position to the desired position over a finite time $T$ horizon, used for a short period of time $\delta<T$, and then recomputed based on the new system state starting at time $t+\delta$ until time $t+T+\delta$. Development and application of receding horizon control (also called model predictive control, or MPC) originated in process control industries where the processes being controlled are often sufficiently slow to permit its implementation. An overview of the evolution of commercially available MPC technology is given in [QB97] and a survey of the state of stability theory of MPC is given in [MRRS00].

\section{Design approach}

The basic philosophy that we propose is illustrated in Figure 3.1. We begin with a nonlinear system, including a description of the constraint set. We linearize this system about a representative equilibrium point and perform a linear control design using standard control design tools. Such a design can provide provably robust performance around the equilibrium point and, more importantly, allows the designer 


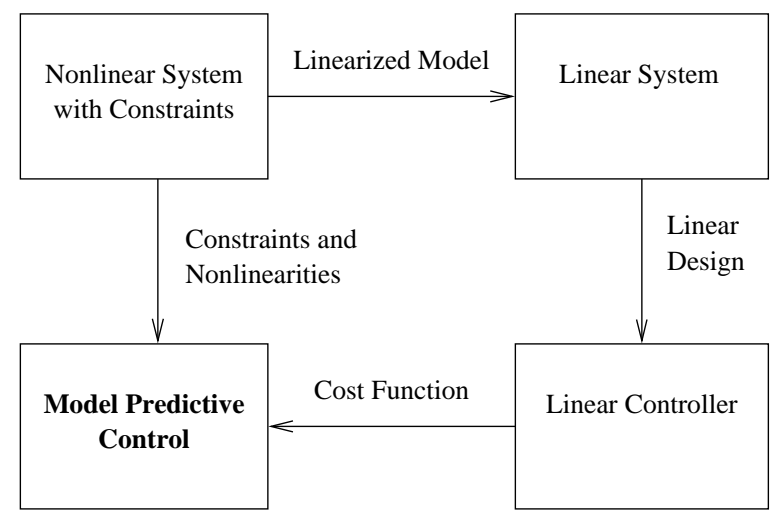

Figure 3.1: Optimization-based control approach.

to meet a wide variety of formal and informal performance specifications through experience and the use of sophisticated linear design tools.

The resulting linear control law then serves as a specification of the desired control performance for the entire nonlinear system. We convert the control law specification into a receding horizon control formulation, chosen such that for the linearized system, the receding horizon controller gives comparable performance. However, because of its use of optimization tools that can handle nonlinearities and constraints, the receding horizon controller is able to provide the desired performance over a much larger operating envelope than the controller design based just on the linearization. Furthermore, by choosing cost formulations that have certain properties, we can provide proofs of stability for the full nonlinear system and, in some cases, the constrained system.

The advantage of the proposed approach is that it exploits the power of humans in designing sophisticated control laws in the absence of constraints with the power of computers to rapidly compute trajectories that optimize a given cost function in the presence of constraints. New advances in online trajectory generation serve as an enabler for this approach and their demonstration on representative flight control experiments shows their viability [MFHM05]. This approach can be extended to existing nonlinear paradigms as well, as we describe in more detail below.

An advantage of optimization-based approaches is that they allow the potential for online customization of the controller. By updating the model that the optimization uses to reflect the current knowledge of the system characteristics, the controller can take into account changes in parameters values or damage to sensors or actuators. In addition, environmental models that include dynamic constraints can be included, allowing the controller to generate trajectories that satisfy complex operating conditions. These modifications allow for many state- and environmentdependent uncertainties to including the receding horizon feedback loop, providing potential robustness with respect to those uncertainties.

A number of approaches in receding horizon control employ the use of terminal state equality or inequality constraints, often together with a terminal cost, to ensure closed loop stability. In Primbs et al. [PND99], aspects of a stabilityguaranteeing, global control Lyapunov function (CLF) were used, via state and 
control constraints, to develop a stabilizing receding horizon scheme. Many of the nice characteristics of the CLF controller together with better cost performance were realized. Unfortunately, a global control Lyapunov function is rarely available and often not possible.

Motivated by the difficulties in solving constrained optimal control problems, researchers have developed an alternative receding horizon control strategy for the stabilization of nonlinear systems [JYH01]. In this approach, closed loop stability is ensured through the use of a terminal cost consisting of a control Lyapunov function (defined later) that is an incremental upper bound on the optimal cost to go. This terminal cost eliminates the need for terminal constraints in the optimization and gives a dramatic speed-up in computation. Also, questions of existence and regularity of optimal solutions (very important for online optimization) can be dealt with in a rather straightforward manner.

\section{Inverse Optimality}

The philosophy presented here relies on the synthesis of an optimal control problem from specifications that are embedded in an externally generated controller design. This controller is typically designed by standard classical control techniques for a nominal process, absent constraints. In this framework, the controller's performance, stability and robustness specifications are translated into an equivalent optimal control problem and implemented in a receding horizon fashion.

One central question that must be addressed when considering the usefulness of this philosophy is: Given a control law, how does one find an equivalent optimal control formulation? The paper by Kalman [Kal64] lays a solid foundation for this class of problems, known as inverse optimality. In this paper, Kalman considers the class of linear time-invariant (LTI) processes with full-state feedback and a single input variable, with an associated cost function that is quadratic in the input and state variables. These assumptions set up the well-known linear quadratic regulator (LQR) problem, by now a staple of optimal control theory.

In Kalman's paper, the mathematical framework behind the LQR problem is laid out, and necessary and sufficient algebraic criteria for optimality are presented in terms of the algebraic Riccati equation, as well as in terms of a condition on the return difference of the feedback loop. In terms of the LQR problem, the task of synthesizing the optimal control problem comes down to finding the integrated cost weights $Q_{x}$ and $Q_{u}$ given only the dynamical description of the process represented by matrices $A$ and $B$ and of the feedback controller represented by $K$. Kalman delivers a particularly elegant frequency characterization of this map [Kal64], which we briefly summarize here.

We consider a linear system

$$
\dot{x}=A x+B u \quad x \in \mathbb{R}^{n}, u \in \mathbb{R}^{m}
$$

with state $x$ and input $u$. We consider only the single input, single output case for now $(m=1)$. Given a control law

$$
u=K x
$$


we wish to find a cost functional of the form

$$
J=\int_{0}^{T} x^{T} Q_{x} x+u^{T} Q_{u} u d t+x^{T}(T) P_{T} x(T)
$$

where $Q_{x} \in \mathbb{R}^{n \times n}$ and $Q_{u} \in \mathbb{R}^{m \times m}$ define the integrated cost, $P_{T} \in \mathbb{R}^{n \times n}$ is the terminal cost, and $T$ is the time horizon. Our goal is to find $P_{T}>0, Q_{x}>0$, $Q_{u}>0$, and $T>0$ such that the resulting optimal control law is equivalent to $u=K x$.

The optimal control law for the quadratic cost function (3.2) is given by

$$
u=-R^{-1} B^{T} P(t)
$$

where $P(t)$ is the solution to the Riccati ordinary differential equation

$$
-\dot{P}=A^{T} P+P A-P B R^{-1} B^{T} P+Q
$$

with terminal condition $P(T)=P_{T}$. In order for this to give a control law of the form $u=K x$ for a constant matrix $K$, we must find $P_{T}, Q_{x}$, and $Q_{u}$ that give a constant solution to the Riccati equation (3.3) and satisfy $-R^{-1} B^{T} P=K$. It follows that $P_{T}, Q_{x}$ and $Q_{u}$ should satisfy

$$
\begin{gathered}
A^{T} P_{T}+P_{T} A-P_{T} B Q_{u}^{-1} B^{T} P_{T}+Q=0 \\
-Q_{u}^{-1} B^{T} P_{T}=K .
\end{gathered}
$$

We note that the first equation is simply the normal algebraic Riccati equation of optimal control, but with $P_{T}, Q$, and $R$ yet to be chosen. The second equation places additional constraints on $R$ and $P_{T}$.

Equation (3.4) is exactly the same equation that one would obtain if we had considered an infinite time horizon problem, since the given control was constant and hence $P(t)$ was forced to be constant. This infinite horizon problem is precisely the one that Kalman considered in 1964, and hence his results apply directly. Namely, in the single-input single-output case, we can always find a solution to the coupled equations (3.4) under standard conditions on reachability and observability [Kal64]. The equations can be simplified by substituting the second relation into the first to obtain

$$
A^{T} P_{T}+P_{T} A-K^{T} R K+Q=0 .
$$

This equation is linear in the unknowns and can be solved directly (remembering that $P_{T}, Q_{x}$ and $Q_{u}$ are required to be positive definite).

The implication of these results is that any state feedback control law satisfying these assumptions can be realized as the solution to an appropriately defined receding horizon control law. Thus, we can implement the design framework summarized in Figure 3.1 for the case where our (linear) control design results in a state feedback controller.

The above results can be generalized to nonlinear systems, in which one takes a nonlinear control system and attempts to find a cost function such that the given controller is the optimal control with respect to that cost.

The history of inverse optimal control for nonlinear systems goes back to the early work of Moylan and Anderson [MA73]. More recently, Sepulchre et al. [SJK97] 
showed that a nonlinear state feedback obtained by Sontag's formula from a control Lyapunov function (CLF) is inverse optimal. The connections of this inverse optimality result to passivity and robustness properties of the optimal state feedback are discussed in Jankovic et al. [JSK99]. Most results on inverse optimality do not consider the constraints on control or state. However, the results on the unconstrained inverse optimality justify the use of a more general nonlinear loss function in the integrated cost of a finite horizon performance index combined with a realtime optimization-based control approach that takes the constraints into account.

\section{Control Lyapunov Functions}

For the optimal control problems that we introduce in the next section, we will make use of a terminal cost that is also a control Lyapunov function for the system. Control Lyapunov functions are an extension of standard Lyapunov functions and were originally introduced by Sontag [Son83]. They allow constructive design of nonlinear controllers and the Lyapunov function that proves their stability. A more complete treatment is given in [KKK95].

Consider a nonlinear control system

$$
\dot{x}=f(x, u), \quad x \in \mathbb{R}^{n}, u \in \mathbb{R}^{m} .
$$

Definition 3.1 (Control Lyapunov Function). A locally positive function $V: \mathbb{R}^{n} \rightarrow$ $\mathbb{R}_{+}$is called a control Lyapunov function (CLF) for a control system (3.5) if

$$
\inf _{u \in \mathbb{R}^{m}}\left(\frac{\partial V}{\partial x} f(x, u)\right)<0 \quad \text { for all } x \neq 0 .
$$

In general, it is difficult to find a CLF for a given system. However, for many classes of systems, there are specialized methods that can be used. One of the simplest is to use the Jacobian linearization of the system around the desired equilibrium point and generate a CLF by solving an LQR problem.

As described in Chapter ??, the problem of minimizing the quadratic performance index,

$$
J=\int_{0}^{\infty}\left(x^{T}(t) Q x(t)+u^{T} R u(t)\right) d t \quad \text { subject to } \quad \begin{aligned}
\dot{x} & =A x+B u, \\
x(0) & =x_{0},
\end{aligned}
$$

results in finding the positive definite solution of the following Riccati equation:

$$
A^{T} P+P A-P B R^{-1} B^{T} P+Q=0
$$

The optimal control action is given by

$$
u=-R^{-1} B^{T} P x
$$

and $V=x^{T} P x$ is a CLF for the system.

In the case of the nonlinear system $\dot{x}=f(x, u), A$ and $B$ are taken as

$$
A=\left.\frac{\partial f(x, u)}{\partial x}\right|_{(0,0)} \quad B=\left.\frac{\partial f(x, u)}{\partial u}\right|_{(0,0)}
$$


where the pairs $(A, B)$ and $\left(Q^{\frac{1}{2}}, A\right)$ are assumed to be stabilizable and detectable respectively. The CLF $V(x)=x^{T} P x$ is valid in a region around the equilibrium $(0,0)$, as shown in Exercise 3.1.

More complicated methods for finding control Lyapunov functions are often required and many techniques have been developed. An overview of some of these methods can be found in [Jad01].

\section{Finite Horizon Optimal Control}

We briefly review the problem of optimal control over a finite time horizon as presented in Chapter ?? to establish the notation for the chapter and set some more specific conditions required for receding horizon control. This material is based on $\left[\mathrm{MHJ}^{+} 03\right]$.

Given an initial state $x_{0}$ and a control trajectory $u(\cdot)$ for a nonlinear control system $\dot{x}=f(x, u)$, let $x^{u}\left(\cdot ; x_{0}\right)$ represent the state trajectory. We can write this solution as a continuous curve

$$
x^{u}\left(t ; x_{0}\right)=x_{0}+\int_{0}^{t} f\left(x^{u}\left(\tau ; x_{0}\right), u(\tau)\right) d \tau
$$

for $t \geq 0$. We require that the trajectories of the system satisfy an a priori bound

$$
\|x(t)\| \leq \beta\left(x, T,\|u(\cdot)\|_{1}\right)<\infty, \quad t \in[0, T],
$$

where $\beta$ is continuous in all variables and monotone increasing in $T$ and $\|u(\cdot)\|_{1}=$ $\|u(\cdot)\|_{L_{1}(0, T)}$. Most models of physical systems will satisfy a bound of this type.

The performance of the system will be measured by an integral cost $L: \mathbb{R}^{n} \times$ $\mathbb{R}^{m} \rightarrow \mathbb{R}$. We require that $L$ be twice differentiable $\left(C^{2}\right)$ and fully penalize both state and control according to

$$
L(x, u) \geq c_{q}\left(\|x\|^{2}+\|u\|^{2}\right), \quad x \in \mathbb{R}^{n}, u \in \mathbb{R}^{m}
$$

for some $c_{q}>0$ and $L(0,0)=0$. It follows that the quadratic approximation of $L$ at the origin is positive definite,

$$
\left.\frac{\partial L}{\partial x}\right|_{(0,0)} \geq c_{q} I>0
$$

To ensure that the solutions of the optimization problems of interest are well behaved, we impose some convexity conditions. We require the set $f\left(x, \mathbb{R}^{m}\right) \subset \mathbb{R}^{n}$ to be convex for each $x \in \mathbb{R}^{n}$. Letting $\lambda \in \mathbb{R}^{n}$ represent the co-state, we also require that the pre-Hamiltonian function $\lambda^{T} f(x, u)+L(x, u)=: K(x, u, \lambda)$ be strictly convex for each $(x, \lambda) \in \mathbb{R}^{n} \times \mathbb{R}^{n}$ and that there is a $C^{2}$ function $\bar{u}^{*}$ : $\mathbb{R}^{n} \times \mathbb{R}^{n} \rightarrow \mathbb{R}^{m}$ providing the global minimum of $K(x, u, \lambda)$. The Hamiltonian $H(x, \lambda):=K\left(x, \bar{u}^{*}(x, \lambda), \lambda\right)$ is then $C^{2}$, ensuring that extremal state, co-state, and control trajectories will all be sufficiently smooth $\left(C^{1}\right.$ or better). Note that these conditions are automatically satisfied for control affine $f$ and quadratic $L$.

The cost of applying a control $u(\cdot)$ from an initial state $x$ over the infinite time interval $[0, \infty)$ is given by

$$
J_{\infty}(x, u(\cdot))=\int_{0}^{\infty} L\left(x^{u}(\tau ; x), u(\tau)\right) d \tau .
$$


The optimal cost (from $x$ ) is given by

$$
J_{\infty}^{*}(x)=\inf _{u(\cdot)} J_{\infty}(x, u(\cdot))
$$

where the control function $u(\cdot)$ belongs to some reasonable class of admissible controls (e.g., piecewise continuous). The function $J_{\infty}^{*}(x)$ is often called the optimal value function for the infinite horizon optimal control problem. For the class of $f$ and $L$ considered, it can be verified that $J_{\infty}^{*}(\cdot)$ is a positive definite $C^{2}$ function in a neighborhood of the origin [HO01].

For practical purposes, we are interested in finite horizon approximations of the infinite horizon optimization problem. In particular, let $V(\cdot)$ be a nonnegative $C^{2}$ function with $V(0)=0$ and define the finite horizon cost (from $x$ using $u(\cdot)$ ) to be

$$
J_{T}(x, u(\cdot))=\int_{0}^{T} L\left(x^{u}(\tau ; x), u(\tau)\right) d \tau+V\left(x^{u}(T ; x)\right)
$$

and denote the optimal cost (from $x$ ) as

$$
J_{T}^{*}(x)=\inf _{u(\cdot)} J_{T}(x, u(\cdot)) .
$$

As in the infinite horizon case, one can show, by geometric means, that $J_{T}^{*}(\cdot)$ is locally smooth $\left(C^{2}\right)$. Other properties will depend on the choice of $V$ and $T$.

Let $\Gamma^{\infty}$ denote the domain of $J_{\infty}^{*}(\cdot)$ (the subset of $\mathbb{R}^{n}$ on which $J_{\infty}^{*}$ is finite). It is not too difficult to show that the cost functions $J_{\infty}^{*}(\cdot)$ and $J_{T}^{*}(\cdot), T \geq 0$, are continuous functions on $\Gamma_{\infty}$ [Jad01]. For simplicity, we will allow $J_{\infty}^{*}(\cdot)$ to take values in the extended real line so that, for instance, $J_{\infty}^{*}(x)=+\infty$ means that there is no control taking $x$ to the origin.

We will assume that $f$ and $L$ are such that the minimum value of the cost functions $J_{\infty}^{*}(x), J_{T}^{*}(x), T \geq 0$, is attained for each (suitable) $x$. That is, given $x$ and $T>0$ (including $T=\infty$ when $\left.x \in \Gamma^{\infty}\right)$, there is a $\left(C^{1}\right.$ in $t$ ) optimal trajectory $\left(x_{T}^{*}(t ; x), u_{T}^{*}(t ; x)\right), t \in[0, T]$, such that $J_{T}\left(x, u_{T}^{*}(\cdot ; x)\right)=J_{T}^{*}(x)$. For instance, if $f$ is such that its trajectories can be bounded on finite intervals as a function of its input size, e.g., there is a continuous function $\beta$ such that $\left\|x^{u}\left(t ; x_{0}\right)\right\| \leq$ $\beta\left(\left\|x_{0}\right\|,\|u(\cdot)\|_{L_{1}[0, t]}\right)$, then (together with the conditions above) there will be a minimizing control (cf. [LM67]). Many such conditions may be used to good effect; see [Jad01] for a more complete discussion.

It is easy to see that $J_{\infty}^{*}(\cdot)$ is proper on its domain so that the sub-level sets

$$
\Gamma_{r}^{\infty}:=\left\{x \in \Gamma^{\infty}: J_{\infty}^{*}(x) \leq r^{2}\right\}
$$

are compact and path connected and moreover $\Gamma^{\infty}=\bigcup_{r \geq 0} \Gamma_{r}^{\infty}$. Note also that $\Gamma^{\infty}$ may be a proper subset of $\mathbb{R}^{n}$ since there may be states that cannot be driven to the origin. We use $r^{2}$ (rather than $r$ ) here to reflect the fact that our integral cost is quadratically bounded from below. We refer to sub-level sets of $J_{T}^{*}(\cdot)$ and $V(\cdot)$ using

$$
\Gamma_{r}^{T}:=\text { path connected component of }\left\{x \in \Gamma^{\infty}: J_{T}^{*}(x) \leq r^{2}\right\} \text { containing } 0,
$$

and

$$
\Omega_{r}:=\text { path connected component of }\left\{x \in \mathbb{R}^{n}: V(x) \leq r^{2}\right\} \text { containing } 0 .
$$


These results provide the technical framework needed for receding horizon control.

\subsection{Receding Horizon Control with CLF Terminal Cost}

In receding horizon control, a finite horizon optimal control problem is solved, generating open-loop state and control trajectories. The resulting control trajectory is applied to the system for a fraction of the horizon length. This process is then repeated, resulting in a sampled data feedback law. Although receding horizon control has been successfully used in the process control industry for many years, its application to fast, stability-critical nonlinear systems has been more difficult. This is mainly due to two issues. The first is that the finite horizon optimizations must be solved in a relatively short period of time. Second, it can be demonstrated using linear examples that a naive application of the receding horizon strategy can have undesirable effects, often rendering a system unstable. Various approaches have been proposed to tackle this second problem; see [MRRS00] for a comprehensive review of this literature. The theoretical framework presented here also addresses the stability issue directly, but is motivated by the need to relax the computational demands of existing stabilizing RHC formulations.

Receding horizon control provides a practical strategy for the use of information from a model through on-line optimization. Every $\delta$ seconds, an optimal control problem is solved over a $T$ second horizon, starting from the current state. The first $\delta$ seconds of the optimal control $u_{T}^{*}(\cdot ; x(t))$ is then applied to the system, driving the system from $x(t)$ at current time $t$ to $x_{T}^{*}(\delta, x(t))$ at the next sample time $t+\delta$ (assuming no model uncertainty). We denote this receding horizon scheme as $\mathcal{R} \mathcal{H}(T, \delta)$.

In defining (unconstrained) finite horizon approximations to the infinite horizon problem, the key design parameters are the terminal cost function $V(\cdot)$ and the horizon length $T$ (and, perhaps also, the increment $\delta$ ). We wish to characterize the sets of choices that provide successful controllers.

It is well known (and easily demonstrated with linear examples), that simple truncation of the integral (i.e., $V(x) \equiv 0$ ) may have disastrous effects if $T>0$ is too small. Indeed, although the resulting value function may be nicely behaved, the "optimal" receding horizon closed loop system can be unstable.

A more sophisticated approach is to make good use of a suitable terminal cost $V(\cdot)$. Evidently, the best choice for the terminal cost is $V(x)=J_{\infty}^{*}(x)$ since then the optimal finite and infinite horizon costs are the same. Of course, if the optimal value function were available there would be no need to solve a trajectory optimization problem. What properties of the optimal value function should be retained in the terminal cost? To be effective, the terminal cost should account for the discarded tail by ensuring that the origin can be reached from the terminal state $x^{u}(T ; x)$ in an efficient manner (as measured by $L$ ). One way to do this is to use an appropriate control Lyapunov function, which is also an upper bound on the cost-to-go.

The following theorem shows that the use of a particular type of CLF is in fact effective, providing rather strong and specific guarantees. 
Theorem 3.1. [JYH01] Suppose that the terminal cost $V(\cdot)$ is a control Lyapunov function such that

$$
\min _{u \in \mathbb{R}^{m}}(\dot{V}+L)(x, u) \leq 0
$$

for each $x \in \Omega_{r_{v}}$ for some $r_{v}>0$. Then, for every $T>0$ and $\delta \in(0, T]$, the resulting receding horizon trajectories go to zero exponentially fast. For each $T>0$, there is an $\bar{r}(T) \geq r_{v}$ such that $\Gamma_{\bar{r}(T)}^{T}$ is contained in the region of attraction of $\mathcal{R H}(T, \delta)$. Moreover, given any compact subset $\Lambda$ of $\Gamma^{\infty}$, there is a $T^{*}$ such that $\Lambda \subset \Gamma_{\bar{r}(T)}^{T}$ for all $T \geq T^{*}$.

Theorem 3.1 shows that for any horizon length $T>0$ and any sampling time $\delta \in(0, T]$, the receding horizon scheme is exponentially stabilizing over the set $\Gamma_{r_{v}}^{T}$. For a given $T$, the region of attraction estimate is enlarged by increasing $r$ beyond $r_{v}$ to $\bar{r}(T)$ according to the requirement that $V\left(x_{T}^{*}(T ; x)\right) \leq r_{v}^{2}$ on that set. An important feature of the above result is that, for operations with the set $\Gamma_{\bar{r}(T)}^{T}$, there is no need to impose stability ensuring constraints which would likely make the online optimizations more difficult and time consuming to solve.

Sketch of proof. Let $x^{u}(\tau ; x)$ represent the state trajectory at time $\tau$ starting from initial state $x$ and applying a control trajectory $u(\cdot)$, and let $\left(x_{T}^{*}, u_{T}^{*}\right)(\cdot, x)$ represent the optimal trajectory of the finite horizon, optimal control problem with horizon $T$. Assume that $x_{T}^{*}(T ; x) \in \Omega_{r}$ for some $r>0$. Then for any $\delta \in[0, T]$ we want to show that the optimal $\operatorname{cost} x_{T}^{*}(\delta ; x)$ satisfies

$$
J_{T}^{*}\left(x_{T}^{*}(\delta ; x)\right) \leq J_{T}^{*}(x)-\int_{0}^{\delta} q\left(L\left(x_{T}^{*}(\tau ; x), u_{T}^{*}(\tau ; x)\right) d \tau .\right.
$$

This expression says that solution to the finite-horizon, optimal control problem starting at time $t=\delta$ has cost that is less than the cost of the solution from time $t=0$, with the initial portion of the cost subtracted off.. In other words, we are closer to our solution by a finite amount at each iteration of the algorithm. It follows using Lyapunov analysis that we must converge to the zero cost solution and hence our trajectory converges to the desired terminal state (given by the minimum of the cost function).

To show equation (3.10) holds, consider a trajectory in which we apply the optimal control for the first $T$ seconds and then apply a closed loop controller using a stabilizing feedback $u=-k(x)$ for another $T$ seconds. (The stabilizing compensator is guaranteed to exist since $V$ is a control Lyapunov function.) Let $\left(x_{T}^{*}, u_{T}^{*}\right)(t ; x)$, $t \in[0, T]$ represent the optimal control and $\left(x^{k}, u^{k}\right)\left(t-T ; x_{T}^{*}(T ; x)\right), t \in[T, 2 T]$ represent the control with $u=-k(x)$ applied where $k$ satisfies $(\dot{V}+L)(x,-k(x)) \leq 0$. Finally, let $(\tilde{x}(t), \tilde{u}(t)), t \in[0,2 T]$ represent the trajectory obtained by concatenating the optimal trajectory $\left(x_{T}^{*}, u_{T}^{*}\right)$ with the CLF trajectory $\left(x^{k}, u^{k}\right)$.

We now proceed to show that the inequality (3.10) holds. The cost of using $\tilde{u}(\cdot)$ for the first $T$ seconds starting from the initial state $\left.x_{T}^{*}(\delta ; x)\right), \delta \in[0,, T]$ is given 
by

$$
\begin{aligned}
J_{T}\left(x_{T}^{*}(\delta ; x), \tilde{u}(\cdot)\right)= & \int_{\delta}^{T+\delta} L(\tilde{x}(\tau), \tilde{u}(\tau)) d \tau+V(\tilde{x}(T+\delta)) \\
= & J_{T}^{*}(x)-\int_{0}^{\delta} L\left(x_{T}^{*}(\tau ; x), u_{T}^{*}(\tau ; x)\right) d \tau-V\left(x_{T}^{*}(T ; x)\right) \\
& \quad+\int_{T}^{T+\delta} L(\tilde{x}(\tau), \tilde{u}(\tau)) d \tau+V(\tilde{x}(T+\delta)) .
\end{aligned}
$$

Note that the second line is simply a rewriting of the integral in terms of the optimal $\operatorname{cost} J_{T}^{*}$ with the necessary additions and subtractions of the additional portions of the cost for the interval $[\delta, T+\delta]$. We can how use the bound

$$
L(\tilde{x}(\tau), \tilde{u}(\tau)) \leq \dot{V}(\tilde{x}(\tau), \tilde{u}(\tau), \quad \tau \in[T, 2 T],
$$

which follows from the definition of the CLF $V$ and stabilizing controller $k(x)$. This allows us to write

$$
\begin{aligned}
J_{T}\left(x_{T}^{*}(\delta ; x), \tilde{u}(\cdot)\right) \leq & J_{T}^{*}(x)-\int_{0}^{\delta} L\left(x_{T}^{*}(\tau ; x), u_{T}^{*}(\tau ; x)\right) d \tau-V\left(x_{T}^{*}(T ; x)\right) \\
& -\int_{T}^{T+\delta} \dot{V}(\tilde{x}(\tau), \tilde{u}(\tau)) d \tau+V(\tilde{x}(T+\delta)) \\
= & J_{T}^{*}(x)-\int_{0}^{\delta} L\left(x_{T}^{*}(\tau ; x), u_{T}^{*}(\tau ; x)\right) d \tau-V\left(x_{T}^{*}(T ; x)\right) \\
& -\left.V(\tilde{x}(\tau))\right|_{T} ^{T+\delta}+V(\tilde{x}(T+\delta)) \\
= & J_{T}^{*}(x)-\int_{0}^{\delta} L\left(x_{T}^{*}(\tau ; x), u_{T}^{*}(\tau ; x)\right) .
\end{aligned}
$$

Finally, using the optimality of $u_{T}^{*}$ we have that $J_{T}^{*}\left(x_{T}^{*}(\delta ; x)\right) \leq J_{T}\left(x_{T}^{*}(\delta ; x), \tilde{u}(\cdot)\right)$ and we obtain equation (3.10).

An important benefit of receding horizon control is its ability to handle state and control constraints. While the above theorem provides stability guarantees when there are no constraints present, it can be modified to include constraints on states and controls as well. In order to ensure stability when state and control constraints are present, the terminal cost $V(\cdot)$ should be a local CLF satisfying $\min _{u \in \mathcal{U}} \dot{V}+L(x, u) \leq 0$ where $\mathcal{U}$ is the set of controls where the control constraints are satisfied. Moreover, one should also require that the resulting state trajectory $x^{C L F}(\cdot) \in \mathcal{X}$, where $\mathcal{X}$ is the set of states where the constraints are satisfied. (Both $\mathcal{X}$ and $\mathcal{U}$ are assumed to be compact with origin in their interior). Of course, the set $\Omega_{r_{v}}$ will end up being smaller than before, resulting in a decrease in the size of the guaranteed region of operation (see [MRRS00] for more details).

\subsection{Receding Horizon Control Using Differential Flatness}

In this section we demonstrate how to use differential flatness to find fast numerical algorithms for solving the optimal control problems required for the receding hori- 
zon control results of the previous section. We consider the affine nonlinear control system

$$
\dot{x}=f(x)+g(x) u,
$$

where all vector fields and functions are smooth. For simplicity, we focus on the single input case, $u \in \mathbb{R}$. We wish to find a trajectory of equation (3.11) that minimizes the performance index (3.8), subject to a vector of initial, final, and trajectory constraints

$$
\begin{aligned}
& l b_{0} \leq \psi_{0}\left(x\left(t_{0}\right), u\left(t_{0}\right)\right) \leq u b_{0}, \\
& l b_{f} \leq \psi_{f}\left(x\left(t_{f}\right), u\left(t_{f}\right)\right) \leq u b_{f}, \\
& l b_{t} \leq S(x, u) \leq u b_{t},
\end{aligned}
$$

respectively. For conciseness, we will refer to this optimal control problem as

$$
\min _{(x, u)} J(x, u) \quad \text { subject to } \quad\left\{\begin{array}{l}
\dot{x}=f(x)+g(x) u, \\
l b \leq c(x, u) \leq u b .
\end{array}\right.
$$

\section{Numerical Solution Using Collocation}

A numerical approach to solving this optimal control problem is to use the direct collocation method outlined in Hargraves and Paris [HP87]. The idea behind this approach is to transform the optimal control problem into a nonlinear programming problem. This is accomplished by discretizing time into a grid of $N-1$ intervals

$$
t_{0}=t_{1}<t_{2}<\ldots<t_{N}=t_{f}
$$

and approximating the state $x$ and the control input $u$ as piecewise polynomials $\tilde{x}$ and $\tilde{u}$, respectively. Typically a cubic polynomial is chosen for the states and a linear polynomial for the control on each interval. Collocation is then used at the midpoint of each interval to satisfy equation (3.11). Let $\tilde{x}\left(x\left(t_{1}\right), \ldots, x\left(t_{N}\right)\right)$ and $\tilde{u}\left(u\left(t_{1}\right), \ldots, u\left(t_{N}\right)\right)$ denote the approximations to $x$ and $u$, respectively, depending on $\left(x\left(t_{1}\right), \ldots, x\left(t_{N}\right)\right) \in \mathbb{R}^{n N}$ and $\left(u\left(t_{1}\right), \ldots, u\left(t_{N}\right)\right) \in \mathbb{R}^{N}$ corresponding to the value of $x$ and $u$ at the grid points. Then one solves the following finite dimension approximation of the original control problem (3.13):

$$
\min _{y \in \mathbb{R}^{M}} F(y)=J(\tilde{x}(y), \tilde{u}(y)) \quad \text { subject to }\left\{\begin{array}{l}
\dot{\tilde{x}}-f(\tilde{x}(y))+g(\tilde{x}(y)) \tilde{u}(y)=0, \\
l b \leq c(\tilde{x}(y), \tilde{u}(y)) \leq u b, \\
\forall t=\frac{t_{j}+t_{j+1}}{2} \quad j=1, \ldots, N-1
\end{array}\right.
$$

where $y=\left(x\left(t_{1}\right), u\left(t_{1}\right), \ldots, x\left(t_{N}\right), u\left(t_{N}\right)\right)$, and $M=\operatorname{dim} y=(n+1) N$.

Seywald [Sey94] suggested an improvement to the previous method (see also [Bry99, p. 362]). Following this work, one first solves a subset of system dynamics in equation (3.13) for the the control in terms of combinations of the state and its time derivative. Then one substitutes for the control in the remaining system dynamics and constraints. Next all the time derivatives $\dot{x}_{i}$ are approximated by the finite difference approximations

$$
\dot{\bar{x}}\left(t_{i}\right)=\frac{x\left(t_{i+1}\right)-x\left(t_{i}\right)}{t_{i+1}-t_{i}}
$$


to get

$$
\left.\begin{array}{r}
p\left(\dot{\bar{x}}\left(t_{i}\right), x\left(t_{i}\right)\right)=0 \\
q\left(\dot{\bar{x}}\left(t_{i}\right), x\left(t_{i}\right)\right) \leq 0
\end{array}\right\} \quad i=0, \ldots, N-1 .
$$

The optimal control problem is turned into

$$
\min _{y \in \mathbb{R}^{M}} F(y) \quad \text { subject to } \quad\left\{\begin{array}{l}
p\left(\dot{\bar{x}}\left(t_{i}\right), x\left(t_{i}\right)\right)=0 \\
q\left(\dot{\bar{x}}\left(t_{i}\right), x\left(t_{i}\right)\right) \leq 0
\end{array}\right.
$$

where $y=\left(x\left(t_{1}\right), \ldots, x\left(t_{N}\right)\right)$, and $M=\operatorname{dim} y=n N$. As with the Hargraves and Paris method, this parameterization of the optimal control problem (3.13) can be solved using nonlinear programming.

The dimensionality of this discretized problem is lower than the dimensionality of the Hargraves and Paris method, where both the states and the input are the unknowns. This induces substantial improvement in numerical implementation.

\section{Differential Flatness Based Approach}

The results of Seywald give a constrained optimization problem in which we wish to minimize a cost functional subject to $n-1$ equality constraints, corresponding to the system dynamics, at each time instant. In fact, it is usually possible to reduce the dimension of the problem further. Given an output, it is generally possible to parameterize the control and a part of the state in terms of this output and its time derivatives. In contrast to the previous approach, one must use more than one derivative of this output for this purpose.

When the whole state and the input can be parameterized with one output, the system is differentially flat, as described in Section 1.3. When the parameterization is only partial, the dimension of the subspace spanned by the output and its derivatives is given by $r$ the relative degree of this output [Isi89]. In this case, it is possible to write the system dynamics as

$$
\begin{aligned}
& x=\alpha\left(z, \dot{z}, \ldots, z^{(q)}\right) \\
& u=\beta\left(z, \dot{z}, \ldots, z^{(q)}\right) \\
& \Phi\left(z, \dot{z}, \ldots, z^{n-r}\right)=0
\end{aligned}
$$

where $z \in \mathbb{R}^{p}, p>m$ represents a set of outputs that parameterize the trajectory and $\Phi: \mathbb{R}^{n} \times \mathbb{R}^{m}$ represents $n-r$ remaining differential constraints on the output. In the case that the system is flat, $r=n$ and we eliminate these differential constraints.

Unlike the approach of Seywald, it is not realistic to use finite difference approximations as soon as $r>2$. In this context, it is convenient to represent $z$ using B-splines. B-splines are chosen as basis functions because of their ease of enforcing continuity across knot points and ease of computing their derivatives. A pictorial representation of such an approximation is given in Figure 3.2. Doing so we get

$$
z_{j}=\sum_{i=1}^{p_{j}} B_{i, k_{j}}(t) C_{i}^{j}, \quad p_{j}=l_{j}\left(k_{j}-m_{j}\right)+m_{j}
$$

where $B_{i, k_{j}}(t)$ is the B-spline basis function defined in [dB78] for the output $z_{j}$ with order $k_{j}, C_{i}^{j}$ are the coefficients of the B-spline, $l_{j}$ is the number of knot intervals, 


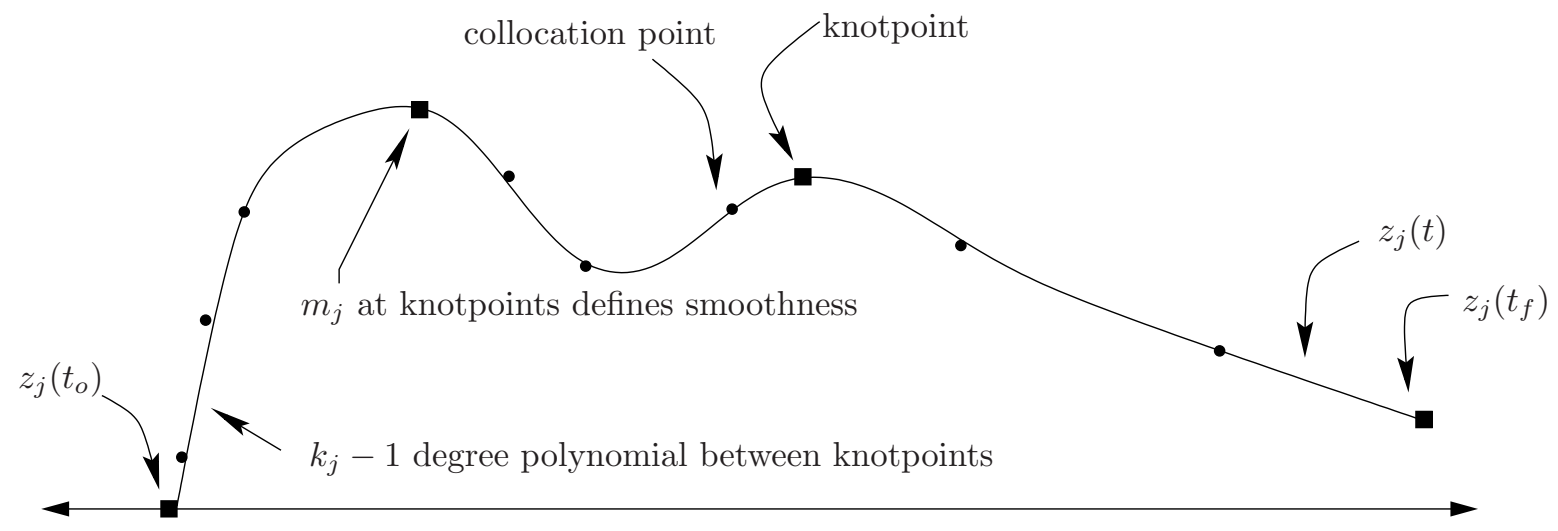

Figure 3.2: Spline representation of a variable.

and $m_{j}$ is number of smoothness conditions at the knots. The set $\left(z_{1}, z_{2}, \ldots, z_{n-r}\right)$ is thus represented by $M=\sum_{j \in\{1, r+1, \ldots, n\}} p_{j}$ coefficients.

In general, $w$ collocation points are chosen uniformly over the time interval $\left[t_{o}, t_{f}\right]$ (though optimal knots placements or Gaussian points may also be considered). Both dynamics and constraints will be enforced at the collocation points. The problem can be stated as the following nonlinear programming form:

$$
\min _{y \in \mathbb{R}^{M}} F(y) \quad \text { subject to } \quad\left\{\begin{array}{c}
\Phi\left(z(y), \dot{z}(y), \ldots, z^{(n-r)}(y)\right)=0 \\
l b \leq c(y) \leq u b
\end{array}\right.
$$

where

$$
y=\left(C_{1}^{1}, \ldots, C_{p_{1}}^{1}, C_{1}^{r+1}, \ldots, C_{p_{r+1}}^{r+1}, \ldots, C_{1}^{n}, \ldots, C_{p_{n}}^{n}\right) .
$$

The coefficients of the B-spline basis functions can be found using nonlinear programming.

A software package called Nonlinear Trajectory Generation (NTG) has been written to solve optimal control problems in the manner described above (see [MMM00] for details). The sequential quadratic programming package NPSOL by [GMSW] is used as the nonlinear programming solver in NTG. When specifying a problem to NTG, the user is required to state the problem in terms of some choice of outputs and its derivatives. The user is also required to specify the regularity of the variables, the placement of the knot points, the order and regularity of the B-splines, and the collocation points for each output.

\subsection{Implementation on the Caltech Ducted Fan}

To demonstrate the use of the techniques described in the previous section, we present an implementation of optimization-based control on the Caltech Ducted Fan, a real-time, flight control experiment that mimics the longitudinal dynamics of an aircraft. The experiment is show in Figure 3.3. 

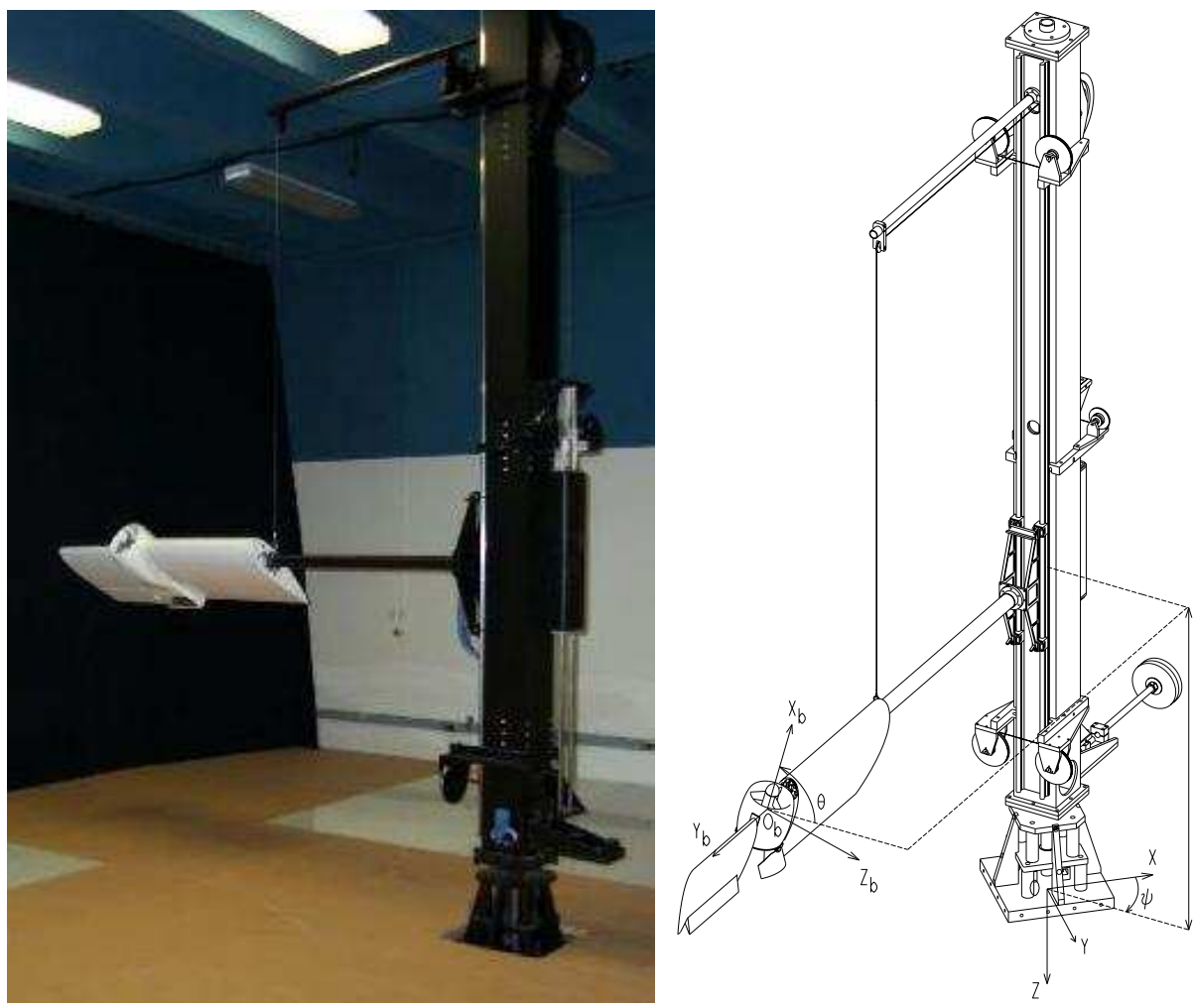

Figure 3.3: Caltech ducted fan.

\section{Description of the Caltech Ducted Fan Experiment}

The Caltech ducted fan is an experimental testbed designed for research and development of nonlinear flight guidance and control techniques for Uninhabited Combat Aerial Vehicles (UCAVs). The fan is a scaled model of the longitudinal axis of a flight vehicle and flight test results validate that the dynamics replicate qualities of actual flight vehicles [MM99].

The ducted fan has three degrees of freedom: the boom holding the ducted fan is allowed to operate on a cylinder, $2 \mathrm{~m}$ high and $4.7 \mathrm{~m}$ in diameter, permitting horizontal and vertical displacements. A counterweight is connected to the vertical axis of the stand, allowing the effective mass of the fan to be adjusted. Also, the wing/fan assembly at the end of the boom is allowed to rotate about its center of mass. Optical encoders mounted on the ducted fan, counterweight pulley, and the base of the stand measure the three degrees of freedom. The fan is controlled by commanding a current to the electric motor for fan thrust and by commanding RC servos to control the thrust vectoring mechanism.

The sensors are read and the commands sent by a DSP-based multi-processor system, comprised of a D/A card, a digital I/O card, two Texas Instruments C40 signal processors, two Compaq Alpha processors, and a high-speed host PC interface. A real-time interface provides access to the processors and I/O hardware. The 
NTG software resides on both of the Alpha processors, each capable of running real-time optimization.

The ducted fan is modeled in terms of the position and orientation of the fan, and their velocities. Letting $x$ represent the horizontal translation, $z$ the vertical translation and $\theta$ the rotation about the boom axis, the equations of motion are given by

$$
\begin{aligned}
m \ddot{x}+F_{X_{a}}-F_{X_{b}} \cos \theta-F_{Z_{b}} \sin \theta & =0, \\
m \ddot{z}+F_{Z_{a}}+F_{X_{b}} \sin \theta-F_{Z_{b}} \cos \theta & =m g_{\mathrm{eff}}, \\
J \ddot{\theta}-M_{a}+\frac{1}{r_{s}} I_{p} \Omega \dot{x} \cos \theta-F_{Z_{b}} r_{f} & =0,
\end{aligned}
$$

where $F_{X_{a}}=D \cos \gamma+L \sin \gamma$ and $F_{Z_{a}}=-D \sin \gamma+L \cos \gamma$ are the aerodynamic forces and $F_{X_{b}}$ and $F_{Z_{b}}$ are thrust vectoring body forces in terms of the lift $(L)$, drag $(D)$, and flight path angle $(\gamma) . I_{p}$ and $\Omega$ are the moment of inertia and angular velocity of the ducted fan propeller, respectively. $J$ is the moment of ducted fan and $r_{f}$ is the distance from center of mass along the $X_{b}$ axis to the effective application point of the thrust vectoring force. The angle of attack $\alpha$ can be derived from the pitch angle $\theta$ and the flight path angle $\gamma$ by

$$
\alpha=\theta-\gamma
$$

The flight path angle can be derived from the spatial velocities by

$$
\gamma=\arctan \frac{-\dot{z}}{\dot{x}} .
$$

The lift $(L)$, drag $(D)$, and moment $(M)$ are given by

$$
L=q S C_{L}(\alpha) \quad D=q S C_{D}(\alpha) \quad M=\bar{c} S C_{M}(\alpha),
$$

respectively. The dynamic pressure is given by $q=\frac{1}{2} \rho V^{2}$. The norm of the velocity is denoted by $V, S$ the surface area of the wings, and $\rho$ is the atmospheric density. The coefficients of lift $\left(C_{L}(\alpha)\right)$, drag $\left(C_{D}(\alpha)\right)$ and the moment coefficient $\left(C_{M}(\alpha)\right)$ are determined from a combination of wind tunnel and flight testing and are described in more detail in [MM99], along with the values of the other parameters.

\section{Real-Time Trajectory Generation}

In this section we describe the implementation of the trajectory generation algorithms by using NTG to generate minimum time trajectories in real time. An LQR-based regulator is used to stabilize the system. We focus in this section on aggressive, forward flight trajectories. The next section extends the controller to use a receding horizon controller, but on a simpler class of trajectories.

\section{Stabilization Around Reference Trajectory}

The results in this section rely on the traditional two degree of freedom design paradigm described in Chapter 1. In this approach, a local control law (inner loop) is used to stabilize the system around the trajectory computed based on a nominal model. This compensates for uncertainties in the model, which are predominantly 
due to aerodynamics and friction. Elements such as the ducted fan flying through its own wake, ground effects and velocity- and angle-of-attack dependent thrust contribute to the aerodynamic uncertainty. Actuation models are not used when generating the reference trajectory, resulting in another source of uncertainty.

Since only the position of the fan is measured, we must estimate the velocities. We use an extended Kalman filter (described in later chapters) with the optimal gain matrix is gain scheduled on the (estimated) forward velocity.

The stabilizing LQR controllers were gain scheduled on pitch angle, $\theta$, and the forward velocity, $\dot{x}$. The pitch angle was allowed to vary from $-\pi / 2$ to $\pi / 2$ and the velocity ranged from 0 to $6 \mathrm{~m} / \mathrm{s}$. The weights were chosen differently for the hoverto-hover and forward flight modes. For the forward flight mode, a smaller weight was placed on the horizontal $(x)$ position of the fan compared to the hover-to-hover mode. Furthermore, the $z$ weight was scheduled as a function of forward velocity in the forward flight mode. There was no scheduling on the weights for hover-tohover. The elements of the gain matrices for each of the controller and observer are linearly interpolated over 51 operating points.

\section{Nonlinear Trajectory Generation Parameters}

We solve a minimum time optimal control problem to generate a feasible trajectory for the system. The system is modeled using the nonlinear equations described above and computed the open loop forces and state trajectories for the nominal system. This system is not known to be differentially flat (due to the aerodynamic forces) and hence we cannot completely eliminate the differential constraints.

We choose three outputs, $z_{1}=x, z_{2}=z$, and $z_{3}=\theta$, which results in a system with one remaining differential constraint. Each output is parameterized with four, sixth order $C^{4}$ piecewise polynomials over the time interval scaled by the minimum time. A fourth output, $z_{4}=T$, is used to represent the time horizon to be minimized and is parameterized by a scalar. There are a total of 37 variables in this optimization problem. The trajectory constraints are enforced at 21 equidistant breakpoints over the scaled time interval.

There are many considerations in the choice of the parameterization of the outputs. Clearly there is a trade between the parameters (variables, initial values of the variables, and breakpoints) and measures of performance (convergence, runtime, and conservative constraints). Extensive simulations were run to determine the right combination of parameters to meet the performance goals of our system.

\section{Forward Flight}

To obtain the forward flight test data, an operator commanded a desired forward velocity and vertical position with joysticks. We set the trajectory update time $\delta$ to 2 seconds. By rapidly changing the joysticks, NTG produces high angle of attack maneuvers. Figure 3.4aa depicts the reference trajectories and the actual $\theta$ and $\dot{x}$ over $60 \mathrm{~s}$. Figure $3.4 \mathrm{~b}$ shows the commanded forces for the same time interval. The sequence of maneuvers corresponds to the ducted fan transitioning from near hover to forward flight, then following a command from a large forward velocity to a large negative velocity, and finally returning to hover. 


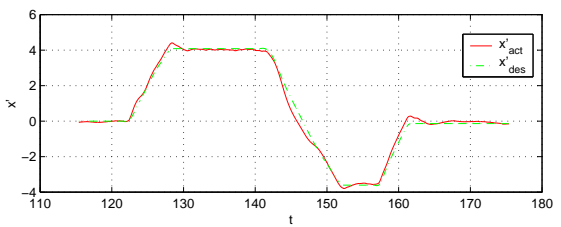

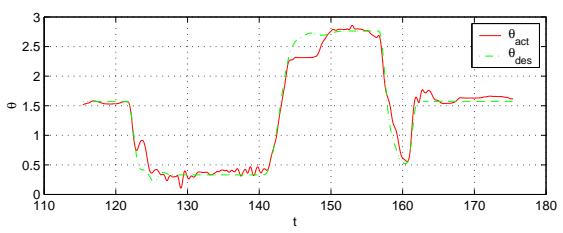

(a) System state

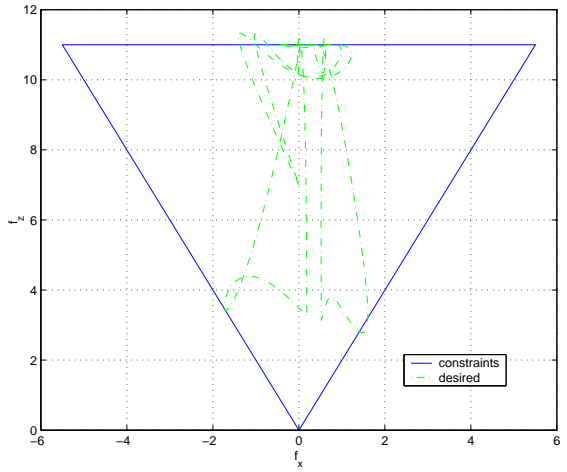

(b) Input forces

Figure 3.4: Forward flight test case: (a) $\theta$ and $\dot{x}$ desired and actual, (b) desired $F_{X_{b}}$ and $F_{Z_{b}}$ with bounds.

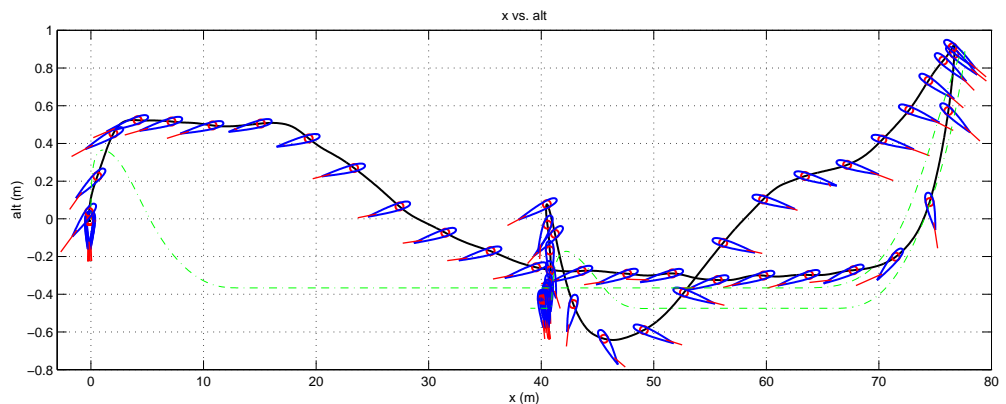

Figure 3.5: Forward flight test case: altitude and $x$ position (actual (solid) and desired (dashed)). Airfoil represents actual pitch angle $(\theta)$ of the ducted fan.

Figure 3.5 is an illustration of the ducted fan altitude and $x$ position for these maneuvers. The air-foil in the figure depicts the pitch angle $(\theta)$. It is apparent from this figure that the stabilizing controller is not tracking well in the $z$ direction. This is due to the fact that unmodeled frictional effects are significant in the vertical direction. This could be corrected with an integrator in the stabilizing controller.

An analysis of the run times was performed for 30 trajectories; the average computation time was less than one second. Each of the 30 trajectories converged to an optimal solution and was approximately between 4 and 12 seconds in length. A random initial guess was used for the first NTG trajectory computation. Subsequent NTG computations used the previous solution as an initial guess. Much improvement can be made in determining a "good" initial guess. Improvement in the initial guess will improve not only convergence but also computation times. 


\section{Receding Horizon Control}

The results of the previous section demonstrate the ability to compute optimal trajectories in real time, although the computation time was not sufficiently fast for closing the loop around the optimization. In this section, we make use of a shorter update time $\delta$, a fixed horizon time $T$ with a quadratic integral cost, and a CLF terminal cost to implement the receding horizon controller described in Section 3.2. We also limit the operation of the system to near hover, so that we can use the local linearization to find the terminal CLF.

We have implemented the receding horizon controller on the ducted fan experiment where the control objective is to stabilize the hover equilibrium point. The quadratic cost is given by

$$
\begin{aligned}
L(x, u) & =\frac{1}{2} \hat{x}^{T} Q \hat{x}+\frac{1}{2} \hat{u}^{T} R \hat{u} \\
V(x) & =\gamma \hat{x}^{T} P \hat{x}
\end{aligned}
$$

where

$$
\begin{aligned}
\hat{x} & =x-x_{e q}=(x, z, \theta-\pi / 2, \dot{x}, \dot{z}, \dot{\theta}) \\
\hat{u} & =u-u_{e q}=\left(F_{X_{b}}-m g, F_{Z_{b}}\right) \\
Q & =\operatorname{diag}\{4,15,4,1,3,0.3\} \\
R & =\operatorname{diag}\{0.5,0.5\}
\end{aligned}
$$

For the terminal cost, we choose $\gamma=0.075$ and $P$ is the unique stable solution to the algebraic Riccati equation corresponding to the linearized dynamics of equation (3.19) at hover and the weights $Q$ and $R$. Note that if $\gamma=1 / 2$, then $V(\cdot)$ is the CLF for the system corresponding to the LQR problem. Instead $V$ is a relaxed (in magnitude) CLF, which achieved better performance in the experiment. In either case, $V$ is valid as a CLF only in a neighborhood around hover since it is based on the linearized dynamics. We do not try to compute off-line a region of attraction for this CLF. Experimental tests omitting the terminal cost and/or the input constraints leads to instability. The results in this section show the success of this choice for $V$ for stabilization. An inner-loop $\mathrm{PD}$ controller on $\theta, \dot{\theta}$ is implemented to stabilize to the receding horizon states $\theta_{T}^{*}, \dot{\theta}_{T}^{*}$. The $\theta$ dynamics are the fastest for this system and although most receding horizon controllers were found to be nominally stable without this inner-loop controller, small disturbances could lead to instability.

The optimal control problem is set-up in NTG code by parameterizing the three position states $(x, z, \theta)$, each with $8 \mathrm{~B}$-spline coefficients. Over the receding horizon time intervals, 11 and 16 breakpoints were used with horizon lengths of 1, 1.5, 2, 3,4 and 6 seconds. Breakpoints specify the locations in time where the differential equations and any constraints must be satisfied, up to some tolerance. The value of $F_{X_{b}}^{\max }$ for the input constraints is made conservative to avoid prolonged input saturation on the real hardware. The logic for this is that if the inputs are saturated on the real hardware, no actuation is left for the inner-loop $\theta$ controller and the system can go unstable. The value used in the optimization is $F_{X_{b}}^{\max }=9 \mathrm{~N}$.

Computation time is non-negligible and must be considered when implementing the optimal trajectories. The computation time varies with each optimization as 


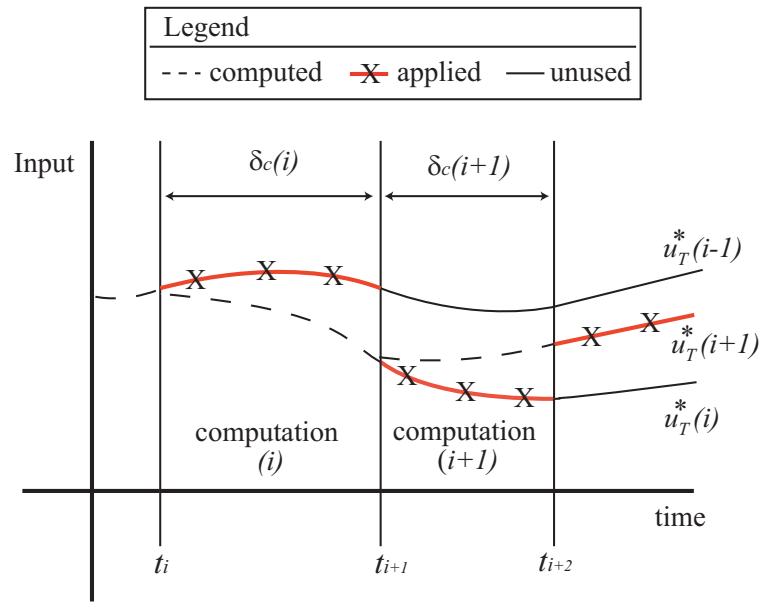

Figure 3.6: Receding horizon input trajectories.

the current state of the ducted fan changes. The following notational definitions will facilitate the description of how the timing is set-up:

$\begin{array}{cl}i & \text { Integer counter of RHC computations } \\ t_{i} & \text { Value of current time when RHC computation } i \text { started } \\ \delta_{c}(i) & \text { Computation time for computation } i \\ u_{T}^{*}(i)(t) & \text { Optimal output trajectory corresponding to computation } \\ & i, \text { with time interval } t \in\left[t_{i}, t_{i}+T\right]\end{array}$

A natural choice for updating the optimal trajectories for stabilization is to do so as fast as possible. This is achieved here by constantly resolving the optimization. When computation $i$ is done, computation $i+1$ is immediately started, so $t_{i+1}=$ $t_{i}+\delta_{c}(i)$. Figure 3.6 gives a graphical picture of the timing set-up as the optimal input trajectories $u_{T}^{*}(\cdot)$ are updated. As shown in the figure, any computation $i$ for $u_{T}^{*}(i)(\cdot)$ occurs for $t \in\left[t_{i}, t_{i+1}\right]$ and the resulting trajectory is applied for $t \in\left[t_{i+1}, t_{i+2}\right]$. At $t=t_{i+1}$ computation $i+1$ is started for trajectory $u_{T}^{*}(i+1)(\cdot)$, which is applied as soon as it is available $\left(t=t_{i+2}\right)$. For the experimental runs detailed in the results, $\delta_{c}(i)$ is typically in the range of $[0.05,0.25]$ seconds, meaning 4 to 20 optimal control computations per second. Each optimization $i$ requires the current measured state of the ducted fan and the value of the previous optimal input trajectories $u_{T}^{*}(i-1)$ at time $t=t_{i}$. This corresponds to, respectively, 6 initial conditions for state vector $x$ and 2 initial constraints on the input vector $u$. Figure 3.6 shows that the optimal trajectories are advanced by their computation time prior to application to the system. A dashed line corresponds to the initial portion of an optimal trajectory and is not applied since it is not available until that computation is complete. The figure also reveals the possible discontinuity between successive applied optimal input trajectories, with a larger discontinuity more likely for longer computation times. The initial input constraint is an effort to reduce such discontinuities, although some discontinuity is unavoidable by this method. Also note that the same discontinuity is present for the 6 open-loop optimal state trajectories generated, again with a likelihood for greater discontinuity for longer 


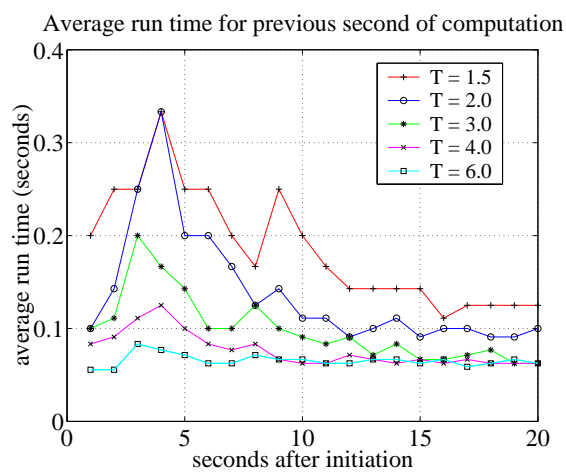

(a) Average run time

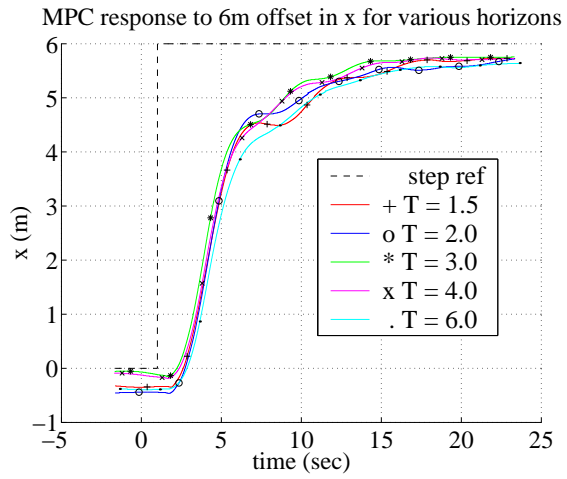

(b) Step responses

Figure 3.7: Receding horizon control: (a) moving one second average of computation time for RHC implementation with varying horizon time, (b) response of RHC controllers to 6 meter offset in $x$ for different horizon lengths.

computation times. In this description, initialization is not an issue because we assume the receding horizon computations are already running prior to any test runs. This is true of the experimental runs detailed in the results.

The experimental results show the response of the fan with each controller to a 6 meter horizontal offset, which is effectively engaging a step-response to a change in the initial condition for $x$. The following details the effects of different receding horizon control parameterizations, namely as the horizon changes, and the responses with the different controllers to the induced offset.

The first comparison is between different receding horizon controllers, where time horizon is varied to be 1.5, 2.0, 3.0, 4.0 or 6.0 seconds. Each controller uses 16 breakpoints. Figure 3.7a shows a comparison of the average computation time as time proceeds. For each second after the offset was initiated, the data correspond to the average run time over the previous second of computation. Note that these computation times are substantially smaller than those reported for real-time trajectory generation, due to the use of the CLF terminal cost versus the terminal constraints in the minimum-time, real-time trajectory generation experiments.

There is a clear trend toward shorter average computation times as the time horizon is made longer. There is also an initial transient increase in average computation time that is greater for shorter horizon times. In fact, the 6 second horizon controller exhibits a relatively constant average computation time. One explanation for this trend is that, for this particular test, a 6 second horizon is closer to what the system can actually do. After 1.5 seconds, the fan is still far from the desired hover position and the terminal cost CLF is large, likely far from its region of attraction. Figure 3.7b shows the measured $x$ response for these different controllers, exhibiting a rise time of 8-9 seconds independent of the controller. So a horizon time closer to the rise time results in a more feasible optimization in this case. 


\subsection{Further Reading}

\section{Exercises}

3.1 Consider a nonlinear control system

$$
\dot{x}=f(x, u)
$$

with linearization

$$
\dot{x}=A x+B u .
$$

Show that if the linearized system is reachable, then there exists a (local) control Lyapunov function for the nonlinear system. (Hint: start by proving the result for a stable system.)

3.2 In this problem we will explore the effect of constraints on control of the linear unstable system given by

$$
\begin{aligned}
& \dot{x}_{1}=0.8 x_{1}-0.5 x_{2}+0.5 u \\
& \dot{x}_{2}=x_{1}+0.5 u
\end{aligned}
$$

subject to the constraint that $|u| \leq a$ where $a$ is a postive constant.

(a) Ignore the constraint $(a=\infty)$ and design an LQR controller to stabilize the system. Plot the response of the closed system from the initial condition given by $x=(1,0)$.

(b) Use SIMULINK or ode45 to simulate the system for some finite value of $a$ with an initial condition $x(0)=(1,0)$. Numerically (trial and error) determine the smallest value of $a$ for which the system goes unstable.

(c) Let $a_{\min }(\rho)$ be the smallest value of $a$ for which the system is unstable from $x(0)=(\rho, 0)$. Plot $a_{\min }(\rho)$ for $\rho=1,4,16,64,256$.

(d) Optional: Given $a>0$, design and implement a receding horizon control law for this system. Show that this controller has larger region of attraction than the controller designed in part (b). (Hint: solve the finite horizon LQ problem analytically, using the bang-bang example as a guide to handle the input constraint.)

3.3 Consider the optimal control problem given in Example ??:

$$
\dot{x}=a x+b u, \quad J=\frac{1}{2} \int_{t_{0}}^{t_{f}} u^{2}(t) d t+\frac{1}{2} c x^{2}\left(t_{f}\right),
$$

where $x \in \mathbb{R}$ is a scalar state, $u \in \mathbb{R}$ is the input, the initial state $x\left(t_{0}\right)$ is given, and $a, b \in \mathbb{R}$ are positive constants. We take the terminal time $t_{f}$ as given and let $c>0$ be a constant that balances the final value of the state with the input required to get to that position. The optimal control for a finite time $T>0$ is derived in Example ??. Now consider the infinite horizon cost

$$
J=\frac{1}{2} \int_{t_{0}}^{\infty} u^{2}(t) d t
$$

with $x(t)$ at $t=\infty$ constrained to be zero. 
(a) Solve for $u^{*}(t)=-b P x^{*}(t)$ where $P$ is the positive solution corresponding to the algebraic Riccati equation. Note that this gives an explicit feedback law $(u=-b P x)$.

(b) Plot the state solution of the finite time optimal controller for the following parameter values

$$
\begin{gathered}
a=2 \quad b=0.5 \quad x\left(t_{0}\right)=4 \\
c=0.1,10 \quad t_{f}=0.5,1,10
\end{gathered}
$$

(This should give you a total of 6 curves.) Compare these to the infinite time optimal control solution. Which finite time solution is closest to the infinite time solution? Why?

Using the solution given in equation (??), implement the finite-time optimal controller in a receding horizon fashion with an update time of $\delta=0.5$. Using the parameter values in part (b), compare the responses of the receding horizon controllers to the LQR controller you designed for problem 1, from the same initial condition. What do you observe as $c$ and $t_{f}$ increase?

(Hint: you can write a MATLAB script to do this by performing the following steps:

(i) set $t_{0}=0$

(ii) using the closed form solution for $x^{*}$ from problem 1 , plot $x(t), t \in\left[t_{0}, t_{f}\right]$ and save $x_{\delta}=x\left(t_{0}+\delta\right)$

(iii) set $x\left(t_{0}\right)=x_{\delta}$ and repeat step (ii) until $x$ is small.)

3.4 In this problem we will explore the effect of constraints on control of the linear unstable system given by

$$
\dot{x}_{1}=0.8 x_{1}-0.5 x_{2}+0.5 u, \quad \dot{x}_{2}=x_{1}+0.5 u,
$$

subject to the constraint that $|u| \leq a$ where $a$ is a postive constant.

(a) Ignore the constraint $(a=\infty)$ and design an LQR controller to stabilize the system. Plot the response of the closed system from the initial condition given by $x=(1,0)$.

(b) Use SIMULINK or ode45 to simulate the system for some finite value of $a$ with an initial condition $x(0)=(1,0)$. Numerically (trial and error) determine the smallest value of $a$ for which the system goes unstable.

(c) Let $a_{\min }(\rho)$ be the smallest value of $a$ for which the system is unstable from $x(0)=(\rho, 0)$. Plot $a_{\min }(\rho)$ for $\rho=1,4,16,64,256$.

(d) Optional: Given $a>0$, design and implement a receding horizon control law for this system. Show that this controller has larger region of attraction than the controller designed in part (b). (Hint: solve the finite horizon LQ problem analytically, using the bang-bang example as a guide to handle the input constraint.) 


\section{Bibliography}

[Bry99] A. E. Bryson. Dynamic optimization. Addison Wesley, 1999.

[dB78] C. de Boor. A Practical Guide to Splines. Springer-Verlag, 1978.

[GMSW] P. E. Gill, W. Murray, M. A. Saunders, and M. Wright. User's Guide for NPSOL 5.0: A Fortran Package for Nonlinear Programming. Systems Optimization Laboratory, Stanford University, Stanford, CA 94305.

[HO01] J. Hauser and H. Osinga. On the geometry of optimal control: The inverted pendulum example. In American Control Conference, 2001.

[HP87] C. Hargraves and S. Paris. Direct trajectory optimization using nonlinear programming and collocation. AIAA J. Guidance and Control, 10:338-342, 1987.

[Isi89] A. Isidori. Nonlinear Control Systems. Springer-Verlag, 2nd edition, 1989.

[Jad01] A. Jadbabaie. Nonlinear Receding Horizon Control: A Control Lyapunov Function Approach. PhD thesis, California Institute of Technology, Control and Dynamical Systems, 2001.

[JSK99] M. Jankovic, R. Sepulchre, and P. V. Kokotović. CLF based designs with robustness to dynamic input uncertainties. Systems Control Letters, 37:45-54, 1999.

[JYH01] A. Jadbabaie, J. Yu, and J. Hauser. Unconstrained receding horizon control of nonlinear systems. IEEE Transactions on Automatic Control, 46, 2001.

[Kal64] R. E. Kalman. When is a linear control system optimal? J. Basic Engrg. Trans. ASME Ser. D, 86:51-60, 1964.

[KKK95] M. Krstić, I. Kanellakopoulos, and P. Kokotović. Nonlinear and Adaptive Control Design. Wiley, 1995.

[LM67] E. B. Lee and L. Markus. Foundations of Optimal Control Theory. Robert E. Krieger Publishing Company, 1967.

[MA73] P. J. Moylan and B. D. O. Anderson. Nonlinear regulator theory and an inverse optimal control problem. IEEE Trans. on Automatic Control, 18(5):460-454, 1973.

$\left[\mathrm{MHJ}^{+} 03\right]$ R. M. Murray, J. Hauser, A. Jadbabaie, M. B. Milam, N. Petit, W. B. Dunbar, and R. Franz. Online control customization via optimization-based control. In T. Samad and G. Balas, editors, Software-Enabled Control: Information Technology for Dynamical Systems. IEEE Press, 2003.

[MM99] M. B. Milam and R. M. Murray. A testbed for nonlinear flight control techniques: The Caltech ducted fan. In Proc. IEEE International Conference on Control and Applications, 1999.

[MMM00] M. B. Milam, K. Mushambi, and R. M. Murray. A computational approach to real-time trajectory generation for constrained mechanical systems. In Proc. IEEE Control and Decision Conference, 2000. 
[MRRS00] D. Q. Mayne, J. B. Rawlings, C. V. Rao, and P. O. M. Scokaert. Constrained model predictive control: Stability and optimality. Automatica, 36(6):789-814, 2000 .

[PND99] J. A. Primbs, V. Nevistić, and J. C. Doyle. Nonlinear optimal control: A control Lyapunov function and receding horizon perspective. Asian Journal of Control, 1(1):1-11, 1999.

[QB97] S. J. Qin and T. A. Badgwell. An overview of industrial model predictive control technology. In J.C. Kantor, C.E. Garcia, and B. Carnahan, editors, Fifth International Conference on Chemical Process Control, pages 232-256, 1997.

[Sey94] H. Seywald. Trajectory optimization based on differential inclusion. J. Guidance, Control and Dynamics, 17(3):480-487, 1994.

[SJK97] R. Sepulchre, M. Jankovic, and P. V. Kokotović. Constructive Nonlinear Control. Springer, London, 1997.

[Son83] E. D. Sontag. A Lyapunov-like characterization of asymptotic controllability. SIAM Journal of Control and Optimization, 21:462-471, 1983. 